\title{
Two scale high gain adaptive control
}

\author{
Jan Willem Polderman \\ Faculty of Mathematical Sciences \\ University of Twente \\ P.O.Box 217, 7500 AE Enschede, The Netherlands \\ E-mail: J.W.Polderman@math.utwente.nl \\ Iven Mareels \\ Department of Electrical and Electronics Engineering \\ The University of Melbourne, VIC 3052 Australia \\ E-mail: i.mareels@unimelb.edu.au
}

\begin{abstract}
Simple adaptive controllers based on high gain output feedback suffer a lack of robustness with respect to bounded disturbances. Existing modifications achieve boundedness of all solutions but introduce solutions that, even in the absence of disturbances, do not achieve regulation. In this paper a new modification that achieves the desired robustness without the side-effect of undesirable solutions is proposed. The modification features two data-driven stability indicators. Intuitively, the first indicator drives the 'closed-loop poles' to the closed right half plane while the latter provides fine-tuning that prevents persistent imaginary axis poles and their associated undesired behaviour.
\end{abstract}

\section{Introduction}

This paper is a sequel to our earlier work on the robustification of high gain adaptive controllers $[5,6]$. The problem that we address is as follows. In simple high gain adaptive controllers, the scalar adaptive feedback gain, say $k$, is driven by the integral of the squared output of the plant $\left(y\right.$, with $\left.\dot{k}=y^{2}\right)$. It can be shown that for linear system with stable zeroes and of relative degree one, this is an effective adaptive algorithm that achieves regulation. It is equally clear, however, that the integration causes robustness problems in the case of measurement errors, noise, or disturbances. A well known fix is the so-called sigma modification [2, chapter 4], [1]. The sigma modification sacrifices regulation (which is not feasible anyway in the presence of arbitrary disturbances) to achieve boundedness, because 
of $\dot{k}=-\sigma k+y^{2}$. However, disappointingly, it does not maintain the stability properties of the original high gain adaptive controller in the noise free case. Examples of possible problems are demonstrated in [6, 4]. In [6] a further modification of the sigma modification is proposed. Concisely, the feedback gain is prevented from decreasing by forcing its derivative to be nonnegative, but the growth prevention is maintained $\dot{k}=\max \left(0,-\sigma k+y^{2}\right)$. This already takes away some of the undesirable properties of the standard sigma modification, indeed it avoids bursting behaviour. However, it does not prevent the feedback gain to converge to a constant for which the closed loop system has eigenvalues on the imaginary axis. This enables nonzero limit cycles in the output. To eliminate these limit cycles, we introduced previously an additional adaptive feedback gain additively combined with the ' $k$ '. This additional feedback gain follows an enumeration of the rationals between zero and one. Crucial is that the additional feedback is kept constant over time intervals of increasing length. This eliminates the possibility of purely imaginary closed loop poles, and hopefully the associated bounded non-zero solutions. There are at least two problems with this approach. Firstly, the additional feedback steps at pre-defined time instants. From a control point of view it would be desirable to let these jump times depend on measurements rather than on time. Secondly, the proof of stability is limited to first and second order systems, and there seems to be no obvious way to extend it to higher order systems. The problem being that it becomes increasingly more complex to exclude the existence of bounded non-zero solutions in higher dimensions.

In the present paper we propose a measurement feedback based modification that allows for a natural proof of stability using more or less standard elements of Lyapunov equations. The idea extends to other types of universal and adaptive controllers.

We use two stability indicators, one based on $\dot{k}=\max \left(0,-\sigma k+y^{2}\right)$, and one based on integrals of the form $\int_{0}^{+T} y^{2}$ over increasingly long intervals $T$. Each time the latter exceeds a prescribed limit the integral is reset, and the adaptive feedback gain is adjusted. The applied adaptive feedback gain consists of two parts. The first part is taken to be equal to the value of the first stability indicator at the start of each interval during which the second stability indicator is constant. The second part is the next rational number taken from an enumeration of all the rationals in $(0,1)$. The feedback gain is the sum of the two parts. An interesting feature of this construction is that the feedback gain is piecewise constant. Not only does this simplify the mathematical analysis significantly, it is also interesting from a control point of view.

The rationale and intuition behind the construction of the feedback gain as the sum of a 'global' and a 'local $\mathcal{L}_{2}$ ' norm driven stability indicator lie in the fact that asymptotic stability of a time invariant linear system is equivalent to $\mathcal{L}_{2}$ stability. The global stability indicator is able to detect instability in that it drives the feedback gain to a value where at least all closed-loop poles are in the closed right half plane. The local stability indicator then fine tunes the feedback gain so that no imaginary axis poles can persist.

The paper is organized as follows. In Section 2 the adaptive control problem is stated. 
In Section 3 the undesirable elements in the classical adaptive algorithm are identified and removed in a step by step process, which illustrates the reasoning and the salient issues. Section 4 presents the completed algorithm with its analysis. Finally, in Section 5 some final observations are gathered.

\section{Problem statement}

Consider SISO systems described by

$$
p\left(\frac{d}{d t}\right) y=q\left(\frac{d}{d t}\right) u
$$

The $p(\xi), q(\xi)$ are polynomials with real coefficients. The scalar signals $u, y$ are the system input and output respectively.

The standing assumptions are:

A1 The polynomial $p(\xi)$ is monic, and the degree of $q(\xi)$ is precisely one less than the degree of $p(\xi)$.

A2 The system defined by (2.1) is controllable, i.e., $p(\xi)$ and $q(\xi)$ have no nontrivial common factors (see [7, Chapter 5]).

A3 The system is minimum phase, i.e., $q(\xi)$ has all its roots in the open left half plane.

A4 The high-frequency gain, the coefficient of the leading term of $q(\xi)$ is positive.

Observe that the actual coefficients of the polynomials $p(\xi), q(\xi)$ as well as their degree are not available for control design.

The control objective is to design a single output feedback law that regulates any one system in the above class, and which is robust with respect to bounded disturbances.

It is a well established fact that systems like (2.1), under the stated assumptions, can be stabilized by constant scalar output feedback $u=-k y$, provided $k$ is sufficiently large.

To ensure that $k$ grows beyond the bound after which the system is asymptotically stable, $k$ is adapted by the truncated $\mathcal{L}_{2}$ norm of the output via $\frac{d}{d t} k=y^{2}$. See [3, Chapter 6] for a self-contained analysis of the resulting adaptive control system. An annoying feature of the adaptation law $\frac{d}{d t} k=y^{2}$ is that the slightest persistent error in the output, e.g. caused by measurement noise, results in unbounded $k$. To avoid this, it has been proposed, see e.g. [2, chapter 4], [1] and the references therein, to incorporate a damping term in the update law:

$$
\frac{d}{d t} k=-\sigma k+y^{2}
$$

Here $\sigma$ is a (small) positive constant. This is usually referred to as the sigma modification. One effect of this modification is clear: bounded $y$ yields bounded $k$. Where the unmodified 
algorithm has an infinite number of equilibria, namely $(y, k)=(0, \bar{k})$ with $\bar{k}$ arbitrary, the sigma modification reduces the set of equilibria to at most three. But where the $\omega$ limit set of the unmodified algorithm consists only of equilibria, the modified algorithm has a much more complicated $\omega$ limit set, on which regulation is not achieved. For instance in the first order case, if the system is unstable, there are three equilibria. Moreover two of these equilibria (the locally attractive ones) correspond to nonzero output so that even in the absence of measurement and control errors the output may not converge to zero. In particular for the case that the controlled system is not asymptotically stable for $k=0$, undesirable behavior may result. Intuitively, for as long as $y^{2}$ is large, $k$ will grow until it reaches a value for which the system stabilizes. Consequently $y^{2}$ will start to decrease. As soon as $-\sigma k$ dominates $y^{2}, k$ will decrease so far as to destabilize the linear system and $y$ will grow again. Depending on the balance between the stabilizing effect of the adaptive gain and the destabilizing effect of the linear plant different solutions may persist. For a detailed study of the nonlinear behavior of the system $(2.1,2.2)$ the reader is referred to [4].

Essentially the problem with the sigma modification is that the damping factor not only prevents $k$ from growing unbounded, it is also responsible for a destabilizing effect as soon as $y^{2}$ becomes small, and this fuels the existence of non-trivial and undesirable solutions. The aim of the present paper is to propose a further modification that eliminates these undesirable solutions while maintaining the robustness properties with respect to measurement errors.

\section{Background and motivation}

The first step is to remove the destabilizing effect. This is easily achieved by preventing $k$ to decrease, whilst limiting the growth as before. So instead of (2.2) consider

$$
\frac{d}{d t} k=\max \left(0,-\sigma k+y^{2}\right)
$$

The effect of the maximization is clear. Since $\frac{d}{d t} k$ is always non-negative, $k$ can never return to a lower and possibly destabilizing, value. Admittedly, $k$ can, due to transients (initial conditions) become larger than absolutely required or even desired for stabilization. The above modification disables the possibility of recovering from that effect. It can also be verified that even when disturbances are present all solutions remain bounded.

Unfortunately, (3.3) does not eliminate all difficulties. Examples illustrating the type of solutions that may exist can be found in [5]. Although (3.3) simplifies the $\omega$-limit set considerably, as compared to (2.2), it is not trivial as desired. Indeed if, for some reason, the output $y$ remains bounded, then the maximization in the update law (3.3) for $k$ enables a dead zone for $k$. Suppose for example that $k(t)=k^{*}$ constant and such that for this feedback gain the controlled system has eigenvalues on the imaginary axis. Then $y(t)$ can oscillate indefinitely if $y^{2}(t)<\sigma k^{*}$. Example 1.1 in [5] illustrates this. So (3.3) is useful in that it simplifies the $\omega$ limit set, but a further modification is needed to eliminate these 'imaginary 
axis eigenvalues' in the closed loop system. It follows from the analysis that other nonlinear phenomena are automatically excluded by this further modification.

A further modification to this effect was proposed in [5]. The intuitive idea is as follows. If the limiting value of the feedback gain would give rise to imaginary axis poles in the controlled system, then this limiting $k$ value should somehow be perturbed to avoid these. This is, however, a nontrivial action. For one, it is not feasible to decide when $k$ is close to its limiting value. Moreover, how does one find out whether the controlled system has (excited) imaginary axis modes. Finally, perturbing the feedback gain without knowing whether or not it is desirable could again lead to a potentially unbounded gain in the presence of disturbances. The idea put forward in [5] is to adjust the feedback gain only during certain carefully chosen intervals of time. Moreover the perturbation must be sufficiently rich to be able to eliminate the existence of imaginary axis poles for any system in the whole class under consideration. This lead to an adaptive feedback law of the following form:

$$
u(t)= \begin{cases}(-k(t)-1) y(t) & t \in I_{2 j} \\ (-k(t)+1) y(t) & t \in I_{2 j+1}\end{cases}
$$

where $\left\{I_{j}\right\}_{j \in \mathbb{N}}$ is a partition of $\mathbb{R}_{+}$. The rationale behind (3.4) is that if $k(t)$ tends to a limit $\bar{k}$ for which $p(\xi)+(\bar{k}+1) q(\xi)$ has roots on the imaginary axis, then $p(\xi)+(\bar{k}-1) q(\xi)$ has no imaginary axis poles. Alternatively, if $p(\xi)+(\bar{k}-1) q(\xi)$ has imaginary axis poles, then $p(\xi)+(\bar{k}+1) q(\xi)$ has not. Obviously, this modification can only work for second order systems. For it could very well happen that in higher order cases that both $p(\xi)+(\bar{k} \pm$ 1) $q(\xi)$ have imaginary axis poles. In fact, if $p$ be a polynomial of order $n$, the polynomials $p(\xi)+k q(\xi), k \in \mathbb{R}$ may have imaginary axis zeroes for at most $n$ distinct values of $k$. The following results make this precise.

Theorem 3.1. Let $n \in \mathbb{N}$ be even and $q(\xi) \in \mathbb{R}[\xi]$ of degree at most $n-1$. Define $N=n / 2$. Let $\omega_{j} \in \mathbb{R}, j=1, \ldots, N$, be $N$ distinct nonzero real scalars and $k_{1}, \ldots, k_{N}$ be arbitrary real scalars. There exists a polynomial $p(\xi) \in \mathbb{R}[\xi]$, monic of degree $n$ such that

$$
p\left(\mathrm{i} \omega_{j}\right)+k_{j} q\left(\mathrm{i} \omega_{j}\right)=0 \text { and } p\left(-\mathrm{i} \omega_{j}\right)+k_{j} q\left(-\mathrm{i} \omega_{j}\right)=0
$$

Proof. With

$$
V=\left[\begin{array}{cccc}
1 & \mathrm{i} \omega_{1} & \cdots & \left(\mathrm{i} \omega_{1}\right)^{n-1} \\
1 & -\mathrm{i} \omega_{1} & \cdots & \left(-\mathrm{i} \omega_{1}\right)^{n-1} \\
\vdots & \vdots & & \vdots \\
\vdots & \vdots & & \vdots \\
1 & \mathrm{i} \omega_{N} & \cdots & \left(\mathrm{i} \omega_{N}\right)^{n-1} \\
1 & -\mathrm{i} \omega_{N} & \cdots & \left(-\mathrm{i} \omega_{N}\right)^{n-1}
\end{array}\right]
$$


the equations (3.5) become:

$$
V\left[\begin{array}{c}
p_{0} \\
\vdots \\
\vdots \\
\vdots \\
p_{n-1}
\end{array}\right]=\left[\begin{array}{c}
-\left(\mathrm{i} \omega_{1}\right)^{n}-k_{1} q\left(\mathrm{i} \omega_{1}\right) \\
-\left(-\mathrm{i} \omega_{1}\right)^{n}-k_{1} q\left(-\mathrm{i} \omega_{1}\right) \\
\vdots \\
\vdots \\
-\left(-\mathrm{i} \omega_{N}\right)^{n}-k_{N} q\left(\mathrm{i} \omega_{N}\right) \\
-\left(-\omega_{N}\right)^{n}-k_{N}\left(-\mathrm{i} \omega_{N}\right)
\end{array}\right]
$$

Observe that $V$ is by construction a nonsingular Vandermonde matrix so that $p(\xi)$ is uniquely defined. As replacing the coefficients of $p(\xi)$ by their complex conjugates also yields a solution, it follows by uniqueness of solutions that the coefficients of $p(\xi)$ must be real.

Theorem 3.2. Let $p(\xi) \in \mathbb{R}[\xi]$ be monic of degree $n$ and $q(\xi) \in \mathbb{R}[\xi]$ be a polynomial of degree $n-1$. Assume that there exists no $\lambda=\mathrm{i} \omega, \omega \in \mathbb{R}$, such that $p(\lambda)=q(\lambda)=0$. The number of distinct values $k$ for which the polynomial $p(\xi)+k q(\xi)$ has zeroes on the imaginary axis does not exceed $n$.

Proof. Let $\lambda=\mathrm{i} \omega, \omega \in \mathbb{R}$ be such that there exists a real $k$ with $p(\lambda)+k q(\lambda)=0$, and thus also $p(-\lambda)+k q(-\lambda)=0$. Because there exists no $\lambda$ such that $p(\lambda)=q(\lambda)=0, k$ may be eliminated to obtain the condition $p(\lambda) q(-\lambda)-p(-\lambda) q(\lambda)=0$. Which can be expanded as $(-1)^{n-1} 2 q_{n-1} \lambda^{2 n-1}+\cdots+2\left(p_{1} q_{0}+p_{0} q_{1}\right) \lambda=0$. It follows that $\lambda=0$ yields a possible solution for $k$. Furthermore, since by assumption $q_{n-1} \neq 0$, there can be at most $n-1$ conjugate pairs $\lambda$ for which $p(\lambda) q(-\lambda)-p(-\lambda) q(\lambda)=0$. Each such pair corresponds to at most one distinct value for $k$ so that $p(\lambda)+k q(\lambda)=0$. This proves the statement.

A generalization of (3.4) that works for systems of arbitrary order must therefore involve an infinite number of variations (rather than just two) of $k$. At the same time the variations should be bounded to keep the total feedback gain, $k$ +variations, bounded. One possibility to achieve this is to use an enumeration of the rationals in a bounded interval, say $(0,1)$. So, let $\left\{a_{j}\right\}_{j \in \mathbb{N}}$ be an enumeration of the rational numbers between zero and one. Define the adaptive feedback law as follows:

$$
u(t)=\left(-k(t)-a_{j}\right) y(t) \quad t \in I_{j}
$$

where again $\left\{I_{j}\right\}_{j \in \mathbb{N}}$ is a partition of $\mathbb{R}_{+}$. The intervals $I_{j}$ should grow sufficiently fast in length so as to enable the detection of stability/instability in the controlled system resulting from $u(t)=\left(-k\left(t_{j}\right)+a_{j}\right) y(t)$. This was further worked out in [6]. In the next section we propose a different modification. Instead of (3.8) we take

$$
u(t)=\left(-k\left(t_{j}\right)-a_{j}\right) y(t) \quad t \in I_{j} .
$$

The difference with (3.8) is that during the time interval $I_{j}$ we use $k\left(t_{j}\right)$ rather than $k(t)$. Also the intervals $I_{j}$ will be determined by output measurements (feedback) rather than by off-line determined time instants (open loop). 


\section{Main result}

Motivated by the discussions in the previous sections we are now ready to present the algorithm in its final form. For clarity of presentation we repeat some of the earlier formulas.

The system (2.1) is given, in state space form, by

$$
\frac{d}{d t} x=A x+b u \quad y=c x
$$

with $(A, c)$ in standard observable form. Let

$$
\begin{aligned}
t_{0} & =0 \\
t_{j+1} & =\min \left\{t \geq t_{j}+1 \mid \int_{t_{j}}^{t} y(\tau)^{2} d \tau \geq j\right\} \\
I_{j} & =\left[t_{j}, t_{j+1}\right) \\
\frac{d}{d t} k & =\max \left(0,-\sigma k+y^{2}\right) \\
\left\{a_{j}\right\}_{j \in \mathbb{N}} & \text { an enumeration of } \mathbb{Q} \cap[0,1] \\
k_{1}(t) & =k\left(t_{j}\right) \quad k_{2}(t)=a_{j} \text { for } t \in I_{j} \\
u(t) & =-\left(k_{1}(t)+k_{2}(t)\right) y(t)
\end{aligned}
$$

It is understood that the $t_{j+1}=\infty$ if the threshold $j$ is never reached. We have the following preliminary result.

Theorem 4.1. Consider the controlled system (4.10,4.11). For every initial condition $\left(x_{0}, k_{0}\right)$ the solution of $(4.10,4.11)$ with $(x(0), k(0))=\left(x_{0}, k_{0}\right)$ exists for all $t \geq 0$, and is unique. Furthermore $x$ and $k_{1}$ are bounded.

Proof. The proof of uniqueness and existence of solution is completely analogous to the case treated in [3, Chapter 6] and is therefore skipped.

Boundedness of $k_{1}$ is readily obtained by contradiction. Suppose that $k_{1}$ is unbounded. Then $k_{1}(t)+k_{2}(t)$ passes the point beyond which any time-varying gain stabilizes the system, see [3, Chapter 6]. Therefore within finite time $-\sigma k(t)+y(t)^{2}<0$ holds. Hence $k$ is bounded and $k_{1}$ is also bounded. Since $k_{1}$ is monotonically non-decreasing and bounded it converges to a limiting value, say $\bar{k}_{1}$.

Boundedness of $y$ also follows by contradiction. Assume that $y$ is not bounded. Since it is the output of a linear system with bounded coefficients, the total length of the time intervals during which $|y|$ is above any given positive constant is infinity. Therefore $y$ is not square integrable. It follows that all switching times $t_{j}$ are finite. As a consequence the adaptive law (4.11b) drives $k$ to infinity. Since $k_{1}(t)=k\left(t_{j}\right.$ for $t \in I_{j}$, and since all switching times $t_{j}$ are finite, it follows that also $k_{1}(t)$ diverges to infinity. This contradicts the boundedness of $k_{1}$, and thus $y$ must be bounded. 
The boundedness of $x$ is achieved as follows. The differential equation (4.10,4.11e) may be re-written as $\frac{d}{d t} x(t)=\left(A+b c\left(k\left(t_{j}\right)+a_{j}\right)\right) x(t)$, for $t \in I_{j}$. Because of observability of $(A, c)$ and the boundedness of $k$, there exist vectors $\ell_{j} \in \mathbb{R}^{1 \times n}$, bounded as a function of $j$, such that $A+b c\left(k\left(t_{j}\right)+a_{j}\right)-\ell_{j} c=: M$ is a time-invariant Hurwitz matrix. Thus $\frac{d}{d t} x(t)=M x(t)+\ell_{j} y(t)$ for $t \in I_{j}$, and thus $x$ is bounded as the state of an asymptotically stable system with bounded input.

Theorem 4.1 shows that the output and its derivatives are bounded. The next step is, of course, the proof that $x$ actually converges to zero. To this end we make use of the following technical Lemma.

Lemma 4.1. Let $\mathcal{A}=\left\{A_{i}\right\}_{i \in \mathbb{N}}$ be a bounded set of matrices in $\mathbb{R}^{n \times n}$. Assume that there exists $\epsilon>0$ such that for all $i$ the matrix $A_{i}$ has no eigenvalues with real part in the interval $(-\epsilon, \epsilon)$. Consider the systems

$$
\frac{d}{d t} x_{i}=A_{i} x_{i}, x_{i}(0)=x_{i, 0}
$$

Furthermore assume that there exist positive constants $T_{i}, M_{i}, i \in \mathbb{N}$ such that

$$
\int_{0}^{T_{i}} x_{i}(t)^{\mathrm{T}} x_{i}(t) d t=M_{i} .
$$

Suppose that there exists a positive constant $K$ such that for all $i$ and for all $t \geq 0$ there holds

$$
\left|x_{i}(t)\right| \leq K
$$

Then there exists a constant $M$ such that for all $i$ :

$$
M_{i} \leq M
$$

Proof. Suppose that $M_{i}$ is not bounded. There exist a bi-bounded ${ }^{1}$ sequence of nonsingular matrices $S_{i}$ such that

$$
S_{i} A_{i} S_{i}^{-1}=\left[\begin{array}{cc}
A_{i, \mathrm{~s}} & 0 \\
0 & A_{i, \mathrm{u}}
\end{array}\right]
$$

with $A_{i, \mathrm{~s}}$ and $-A_{i, \mathrm{u}}$ Hurwitz. This is can be seen as follows. Firstly, there exist orthonormal matrices $U_{i}$ such that $U_{i} A U_{i}^{\mathrm{T}}$ is a real Schur decomposition:

$$
U_{i} A_{i} U_{i}^{\mathrm{T}}=\left[\begin{array}{cc}
A_{i, \mathrm{~s}} & \tilde{A}_{12} \\
0 & A_{i, \mathrm{u}}
\end{array}\right] .
$$

Secondly, there exist matrices $W_{i}$ such that

$$
\underbrace{\left[\begin{array}{cc}
I & W_{i} \\
0 & I
\end{array}\right]}_{Y_{i}}\left[\begin{array}{cc}
A_{i, \mathrm{~s}} & \tilde{A}_{12} \\
0 & A_{i, \mathrm{u}}
\end{array}\right] \underbrace{\left[\begin{array}{cc}
I & -W_{i} \\
0 & I
\end{array}\right]}_{Y_{i}^{-1}}=\left[\begin{array}{cc}
A_{i, \mathrm{~s}} & 0 \\
0 & A_{i, \mathrm{u}}
\end{array}\right] .
$$

\footnotetext{
${ }^{1}$ Recall that a sequence of nonsingular matrices is bi-bounded if it is bounded and if the sequence of inverses is bounded.
} 
The $W_{i}$ s satisfy

$$
A_{i, \mathrm{~s}} W_{i}-W_{i} A_{i, \mathrm{u}}=\tilde{A}_{12} .
$$

Now, since the sequences $\left\{A_{i, \mathrm{~s}}\right\}$ and $\left\{A_{i, \mathrm{u}}\right\}$ are bounded and since the real parts of $A_{i, \mathrm{~s}}$ and $-A_{i, \mathrm{u}}$ are smaller than or equal to $-\epsilon$, it follows that also the sequence $\left\{W_{i}\right\}$ is bounded. Of course, $S_{i}$ is now defined as $S_{i}=Y_{i} U_{i}$.

There exist positive definite matrices $P_{i, \mathrm{~s}}, P_{i, \mathrm{u}}$ such that

$$
A_{i, \mathrm{~s}}^{\mathrm{T}} P_{i, \mathrm{~s}}+P_{i, \mathrm{~s}} A_{i, \mathrm{~s}}=-I \quad A_{i, \mathrm{u}}^{\mathrm{T}} P_{i, \mathrm{u}}+P_{i, \mathrm{u}} A_{i, \mathrm{u}}=I
$$

Define $P_{i}=\operatorname{diag}\left(P_{i, \mathrm{~s}}, P_{i, \mathrm{u}}\right)$ and $z_{i}=S_{i} x_{i}$. Then

$$
\begin{aligned}
z_{i}\left(T_{i}\right)^{\mathrm{T}} & P_{i} z_{i}\left(T_{i}\right) \\
= & z_{i}(0)^{\mathrm{T}} P_{i} z_{i}(0) \\
& \quad+\int_{0}^{T_{i}} z_{i, \mathrm{u}}(t)^{\mathrm{T}} z_{i, \mathrm{u}}(t)-z_{i, \mathrm{~s}}(t)^{\mathrm{T}} z_{i, \mathrm{~s}}(t) d t \\
= & z_{i}(0)^{\mathrm{T}} P_{i} z_{i}(0) \\
& \quad+\int_{0}^{T_{i}} z_{i}(t)^{\mathrm{T}} z_{i}(t)-2 z_{i, \mathrm{~s}}(t)^{\mathrm{T}} z_{i, \mathrm{~s}}(t) d t
\end{aligned}
$$

Define

$$
\int_{0}^{T_{i}} z_{i}(t)^{\mathrm{T}} z_{i}(t) d t=\tilde{M}_{i} .
$$

By the relation between $x_{i}$ and $z_{i}$, the boundedness of the sequence $A_{i}$ and the sequence $S_{i}$ it follows that the unboundedness of $M_{i}$ implies the unboundedness of the sequence $\tilde{M}_{i}$. From the boundedness of the sequence $A_{i}$ and the assumption on the spectra of $A_{i}$ it follows that the sequence $P_{i}$ is bounded. Also, the states $z_{i}(t)$ are bounded, uniformly in $i$. Finally, due to the uniform (in $i$ ) stability of the $A_{i}$ s it follows that the contribution of $2 z_{i, \mathrm{~s}}(t)^{\mathrm{T}} z_{i, \mathrm{~s}}(t)$ in (4.21) is bounded, again uniformly in $i$. Therefore the unboundedness of $\int_{0}^{T_{i}} z_{i}(t)^{\mathrm{T}} z_{i}(t) d t$ as an expression in $i$ implies the unboundedness of $z_{i}\left(T_{i}\right)^{\mathrm{T}} P_{i} z_{i}\left(T_{i}\right)$. This is a contradiction and therefore the sequence $M_{i}$ is bounded.

We are now ready to state and prove the main result of the paper.

Theorem 4.2. Consider the controlled system (4.10,4.11). Then, for every $\left(x_{0}, k_{0}\right)$ the solution of $(4.10,4.11)$ with $(x(0), k(0))=\left(x_{0}, k_{0}\right)$ exists for all $t \geq 0$, is unique and bounded. Moreover $\lim _{t \rightarrow \infty} x(t)=0$.

Proof. Existence, uniqueness, and boundedness of solutions were established in Theorem 4.1. Suppose that $y$ is not square integrable on $[0, \infty)$. Then for all $j$ there holds $t_{j}<\infty$ and moreover $t_{j} \uparrow \infty$ as $j \rightarrow \infty$. Define $A_{j}=A-b c\left(k_{1}\left(t_{j}\right)+a_{j}\right)$. We know that $k\left(t_{j}\right)$ converges, say $\lim _{j \rightarrow \infty} k_{1}\left(t_{j}\right)=k_{1}$. From Theorem 3.2 it follows that $A-b c\left(k_{1}+a\right)$ may have imaginary axis poles for at most $n$ values of $a$. It follows that there exist $\epsilon>0$ and 
sequence $\left\{j_{\ell}\right\}_{\ell \in \mathbb{N}}$ such that $A_{j_{\ell}}$ has no eigenvalues with real parts between $-\epsilon$ and $\epsilon$. Now, apply Lemma 4.1 to the collection of matrices $A_{j_{\ell}}$ to conclude that $x$ is unbounded, but then is also $y$ unbounded (as bounded $y$ implies bounded $x$ ). This contradicts Theorem 4.1 and therefore $y$ is square integrable. By standard reasoning, [3, Chapter 6] (see also the end of the proof of Theorem 4.1) it follows that $y$ and $x$ converge to zero.

\section{Conclusions}

The main result is that there exists a relatively simply adaptive control law that achieves robust regulation for the class of linear systems that can be stabilized via static high gain output feedback. As demonstrated the adaptive algorithm asymptotically stabilizes the plant, and in the presence of disturbances a bounded response is guaranteed. An important feature of the proposed algorithm is that it involves a two stage switched feedback gain where the switching times are generated in true feedback fashion.

\section{References}

[1] P. Ionnnou and P.V. Kokotovic. Instability analysis and improvement for robustness of adaptive control. Automatica, 20:583-594, 1984.

[2] H. Kaufman, I. Barkana, And K. Sobel. Direct adaptive control algorithms, theory and applications. Springer, New York NY, USA, second edition, 1998.

[3] I.M.Y. Mareels And J.W. Polderman. Adaptive Systems: an Introduction. Birkhäuser, Boston, MA, 1996.

[4] I.M.Y. Mareels, S.A. van Gils, J.W. Polderman, and A. Ilchmann. Asymptotic dynamics in adaptive gain control. In Paul M. Frank, editor, Advances in Control, highlights of the 5th European Control Conference, Karlsruhe, Germany, 29-64. Springer, 1999.

[5] J.W. Polderman and I.M.Y. Mareels. High gain adaptive control revisited: first and second order case. In Proc. 38th IEEE Conf. Decision and Control, 3329-3333, Phoenix, AZ, 1999.

[6] J.W. Polderman and I.M.Y. Mareels. Robustification of high gain adaptive control. In Proceedings of the 6th European Control Conference, 2428-2432, Porto, Portugal, 2001.

[7] J.W. Polderman AND J.C. Willems. Introduction to mathematical systems theory: a behavioral approach, volume 26 of Texts in Applied Mathematics. Springer, New York NY, USA, 1997. 